\title{
Lignin provides mechanical support to herbaceous peony (Paeonia lactiflora Pall.) stems
}

\author{
Daqiu Zhao', Yuting Luan', Xing Xia ${ }^{1}$, Wenbo Shi ${ }^{1}$, Yuhan Tang ${ }^{1}$ and Jun Tao ${ }^{1,2}$
}

\begin{abstract}
Stem bending caused by mechanical failure is a major constraint for high-quality herbaceous peony (Paeonia lactiflora Pall.) cut flowers, but little is known about the underlying factors. In this study, two P. lactiflora cultivars, Xixia Yingxue (bending) and Hong Feng (upright), were used to investigate differences in stem bending. The results showed that the stem mechanical strength of Hong Feng was significantly higher than that of Xixia Yingxue, and the thickening of the secondary cell wall and the number of thickened secondary cell wall layers in Hong Feng were significantly higher than those in Xixia Yingxue. Moreover, compared with Xixia Yingxue, Hong Feng showed greater lignification of the cell wall and lignin deposition in the cell walls of the sclerenchyma, vascular bundle sheath and duct. All three types of lignin monomers were detected. The S-lignin, G-lignin, and total lignin contents and the activities of several lignin biosynthesis-related enzymes were higher in Hong Feng than in the other cultivar, and the S-lignin content was closely correlated with stem mechanical strength. In addition, 113,974 full-length isoforms with an average read length of $2106 \mathrm{bp}$ were obtained from the full-length transcriptome of $P$. lactiflora stems, and differential expression analysis was performed based on the comparative transcriptomes of these two cultivars. Ten lignin biosynthesis-related genes, including 26 members that were closely associated with lignin content, were identified, and multiple upregulated and downregulated transcription factors were found to positively or negatively regulate lignin biosynthesis. Consequently, lignin was shown to provide mechanical support to P. lactiflora stems, providing useful information for understanding the formation of $P$. lactiflora stem strength.
\end{abstract}

\section{Introduction}

Stem bending or lodging caused by stem mechanical failure is one of the most important agronomic problems and can cause substantial economic losses associated with the quality and yield of agricultural products. For example, bending of Gerbera jamesonii cut flowers reduces their ornamental value ${ }^{1}$, lodging contributes to poor grain yield and quality of wheat ${ }^{2}$, and mechanical bending induces tension wood formation in Liriodendron tulipifera ${ }^{3}$. Stem mechanical strength is a complex trait influenced by many factors and is closely related to the morphological, structural, and physiological characteristics of stems; increasing

Correspondence: Jun Tao (taojun@yzu.edu.cn)

${ }^{1}$ College of Horticulture and Plant Protection, Yangzhou University, Yangzhou 225009, P.R. China

${ }^{2}$ Joint International Research Laboratory of Agriculture and Agri-Product Safety, The Ministry of Education of China, Yangzhou University, Yangzhou 225009, P.R. China stem mechanical strength can improve resistance to stem bending and lodging ${ }^{4,5}$. Previous studies have shown that morphological characteristics, including stem diameter, stem weight, and internode length, can affect the stem mechanical strength of rye ${ }^{6}$, switchgrass, and miscanthus ${ }^{7}$. Moreover, the anatomy of the stem, which primarily depends on the morphology and distribution of mechanical and transduction tissues and the degree of the development of the xylem, can contribute to stem mechanical strength $^{4}$. In addition, the stem chemical constituents, including cellulose, hemicellulose, and lignin, have an important role in the stem mechanical strength ${ }^{8}$.

Lignin is an integral structural component that is deposited together with cellulose and hemicellulose in the secondary cell walls in vascular plants ${ }^{9}$. Previous studies of sudangrass ${ }^{10}$, chrysanthemum ${ }^{11}$, kodo millet $^{12}$, and rice ${ }^{13}$ have shown that reductions in the lignin content caused the stem mechanical strength to decrease, suggesting that lignin 
is closely tied to stem mechanical strength. Moreover, lignin is composed of guaiacyl, syringyl, and p-hydroxyphenyl units derived from monolignol precursors ( $p$-coumaryl alcohol [the $\mathrm{H}$ unit], coniferyl alcohol [the $\mathrm{G}$ unit], and sinapyl alcohol [the $\mathrm{S}$ unit], respectively ${ }^{14}$. Previous studies showed that the increase in wheat planting density resulted in substantial reductions in S-lignin but no changes in G-lignin, which caused a decrease in the mechanical strength $^{15}$. In studies involving the flexible culm1 mutant of rice and the mechanical bending Liriodendron tulipifera, S-lignin was shown to decrease significantly ${ }^{3,13}$. In addition, enzymes, such as phenylalanine ammonia-lyase (PAL), tyrosine ammonia-lyase (TAL), cinnamate 4-hydroxylase $(\mathrm{C} 4 \mathrm{H})$, coumarate $3^{\prime}$-hydroxylase $\left(\mathrm{C}^{\prime} \mathrm{H}\right)$, caffeic acid $\mathrm{O}$ methyltransferase (COMT), ferulate 5-hydroxylase (F5H), 4-coumarate: CoA ligase (4CL), caffeoyl-CoA O-methyltransferase (CCoAOMT), hydroxycinnamoyl CoA (HCT), cinnamoyl-CoA reductase (CCR), cinnamyl alcohol dehydrogenase (CAD), polyphenol oxidase (PPO), and peroxidase (POD) are involved in catalyzing many steps of the lignin biosynthesis pathway ${ }^{16}$, and significant correlations have been found between the activities of these enzymes and the lignin contents of stems ${ }^{17-19}$.

Herbaceous peony (Paeonia lactiflora Pall.) is a popular ornamental plant species often used to arrange flower borders, flower beds, potted plants, etc. In addition, $P$. lactiflora has also emerged as a high-end cut flower in the international market in recent years and requires thick and upright stems. However, many P. lactiflora cultivars cannot be used as cut flowers because they have elongated stems and droopy flowers. Therefore, studying how the stem mechanical strength of $P$. lactiflora forms is important. To date, some progress has been made in study of $P$. lactiflora stem mechanical strength. For example, Li et al. ${ }^{20,21}$. found that $P$. lactiflora stem mechanical strength was significantly positively correlated with stem diameter, stem weight, and calcium and silicon contents. Subsequently, lignin was found to be the main factor affecting $P$. lactiflora stem mechanical strength $^{22-24}$. Moreover, foliar spraying of calcium and silicon could significantly improve $P$. lactiflora stem mechanical strength by increasing the lignin content ${ }^{25-27}$. However, the relationships between the synthesis, deposition and structure of lignin and the mechanical strength of P. lactiflora stem need to be further studied. To explore the physiological, biochemical, and molecular mechanisms underlying the bending resistance of stems and to clarify the influence of lignin on stem mechanical strength, two P. lactiflora cultivars with different uprightness were used to investigate the differences in their morphological indices and mechanical strength; their secondary cell wall and its lignification; their lignin synthesis, deposition and structure; and their transcriptome sequences (via RNA-seq). The present study was conducted to provide a theoretical basis for understanding the formation of P. lactiflora stem strength.

\section{Materials and methods \\ Plant materials}

Two P. lactiflora cultivars with different uprightness, Xixia Yingxue (bending) and Hong Feng (upright), were used in this study ${ }^{20}$. They were planted in the field of National Germplasm Repository of Yangzhou University, Jiangsu Province, China $\left(32^{\circ} 39^{\prime} \mathrm{N}, 119^{\circ} 42^{\prime} \mathrm{E}\right)$. The top $12 \mathrm{~cm}$ of the stems in the flower-bud stage (S1), unfoldedpetal stage (S2), and the full-bloom stage (S3) were collected, and their morphological indices were determined. Subsequently, the part between the top $5 \mathrm{~cm}$ and top $8 \mathrm{~cm}$ of the stems was fixed in $2.5 \%$ glutaraldehyde solution and FAA fix solution for microstructure, lignification, and lignin deposition observations, and the other part of the stems was immediately frozen in liquid nitrogen and then stored at $-80^{\circ} \mathrm{C}$.

\section{Morphological indices and mechanical strength determination}

Plant height, stem diameter, stem weight, flower diameter and flower weight were measured by a micrometer scale and balance, respectively. Moreover, a universal NK-2 digital force testing device (Zhejiang Hui'er Instrument and Equipment Co., Ltd., China) was used to measure the mechanical strength of the stems.

\section{Secondary cell wall observations}

Secondary cell walls of the stems were observed by environmental scanning electron microscopy (Philips XL-30 ESEM, Holland) and transmission electron microscopy (TEM) (Philips CM100, Holland). The specific details can be found in a study by Zhao et al. ${ }^{28}$.

\section{Lignification and lignin deposition observations}

For paraffin sectioning, 3-4 mm cross-cut stems from the part between the top 5 and $8 \mathrm{~cm}$ were selected and then fixed in FAA fix solution for more than $24 \mathrm{~h}$. The procedure of preparing the paraffin sections was as follows: first, the samples were soaked in mixtures of $50 \%$ ethanol:glycerin $(1: 1, \mathrm{v} / \mathrm{v})$ to soften for $12 \mathrm{~h}$. Then, a gradient of ethanol solutions (50, 70, 90 and 100\%, $30 \mathrm{~min}$ each) was used for sample dehydration, followed by a gradient of mixtures of dimethylbenzene:ethanol (1:3, 1:1, $3: 1, \mathrm{v} / \mathrm{v}, 30 \mathrm{~min}$ each). Finally, mixtures of dimethylbenzene:chloroform $(9: 1, \mathrm{v} / \mathrm{v})$ were used to complete the last dehydration; this repeated twice, and each time lasted for $30 \mathrm{~min}$. Second, each sample was transferred to a $5 \mathrm{~mL}$ centrifuge tube, added to a paraffin:dimethylbenzene mixture $(1: 1, \mathrm{v} / \mathrm{v})$, incubated at $42{ }^{\circ} \mathrm{C}$ for $12 \mathrm{~h}$, and then embedded in pure paraffin. Each sample was then incubated at $42{ }^{\circ} \mathrm{C}$ for $24 \mathrm{~h}$, after which it was embedded in pure paraffin. Each sample was then incubated at $50{ }^{\circ} \mathrm{C}$ for $3 \mathrm{~h}$ and then embedded in pure paraffin; it was subsequently incubated at $60^{\circ} \mathrm{C}$ for $3 \mathrm{~h}$, and replaced by a new 
centrifuge tube and covered using pure paraffin; finally, the sample was incubated at $60^{\circ} \mathrm{C}$ for $2 \mathrm{~h}$, and replaced using pure paraffin three times. Third, each sample was put in a metal mold, after which a plastic mold was used to cover the sample for fixing. Liquid paraffin was slowly added until it overflowed, after which the metal mold was then put on a cooling table until it solidified; finally, the embedded sample was removed. After trimming the paraffin block to a suitable size, each sample was placed on a microtome and sliced. The cut paraffin section was placed in a $40^{\circ} \mathrm{C}$ water bath for expansion, and after it had fully unfolded, it was attached to a glass slide and heated at $45^{\circ} \mathrm{C}$. Finally, the paraffin section was soaked in dimethylbenzene for $10 \mathrm{~min}$, which was repeated once. Afterward, the section was soaked in a mixture of methylbenzene:ethanol $(1: 1, \mathrm{v} / \mathrm{v})$ for $5 \mathrm{~min}$ and a gradient of ethanol solutions (90, 80, 70, and 50\%, 5 min each) was used for rehydration. In addition, the prepared paraffin section was used for subsequent staining and observations.

Based on the prepared paraffin sections, the lignification and lignin deposition were observed using the crystal violet staining method and phloroglucinol staining method, the specific details of which are available in the study by Zhao et al. ${ }^{28}$.

\section{Lignin structure analysis}

Lignin structure was analyzed using Fourier transform infrared (FTIR) and two-dimensional heteronuclear single quantum coherence (2D-HSQC). The lignin powder of the stem was extracted by the high boiling solvent method $^{29}$.

For FTIR, the specific details are available in the study by Zhao et al. ${ }^{28}$. For 2D-HSQC, $20 \mathrm{mg}$ of lignin powder was first dissolved in $0.5 \mathrm{~mL}$ of DMSO-d6. The spectra were then recorded at $25^{\circ} \mathrm{C}$ using a nuclear magnetic resonance spectrometer (Bruker AVANCE 600 , Switzerland). The spectral widths were 3,497 and $18,750 \mathrm{~Hz}$ for the ${ }^{1} \mathrm{H}$ and ${ }^{13} \mathrm{C}$ dimensions, respectively. The number of sampling points in the ${ }^{1} \mathrm{H}$ dimension was 1,024 , the relaxation time was $1.5 \mathrm{~s}$. The number of collected sampling points in the ${ }^{13} \mathrm{C}$ dimension was 256 , and the ${ }^{1} J_{\mathrm{CH}}$ was $145 \mathrm{~Hz}$. Prior to Fourier transformation, the data matrices were zero-filled to 1024 points in the ${ }^{13} \mathrm{C}$ dimension. The central solvent (DMSO) peak was used as an internal chemical shift reference point $\left(\delta_{\mathrm{C}} / \delta_{\mathrm{H}}\right.$ of $39.5 / 2.49)$.

\section{Lignin content measurements}

Resolution of the lignin polymer was performed by alkaline nitrobenzene oxidation. A total of $0.05 \mathrm{~g}$ of dry lignin powder was placed in a $25 \mathrm{~mL}$ polytetrafluoroethylene sealed tank containing $5 \mathrm{~mL}$ of $\mathrm{NaOH}$ solution $(2 \mathrm{~mol} / \mathrm{L})$ and $0.5 \mathrm{~mL}$ of nitrobenzene and reacted at $170^{\circ} \mathrm{C}$ for $3.5 \mathrm{~h}$. After cooling, the reaction mixture was transferred to a $100 \mathrm{~mL}$ erlenmeyer flask, and $0.2 \mathrm{~mL}$ of ethyl vanillin solution $(4 \mathrm{mg} / \mathrm{mL})$ was added as an internal standard. The reaction mixture was extracted three times using $30 \mathrm{~mL}$ of a dichloromethane:ethyl acetate mixture $(1: 1, \mathrm{v}: \mathrm{v})$, and the $\mathrm{pH}$ of the resulting aqueous phase was adjusted to $3.0-4.0$ with $6 \mathrm{~mol} / \mathrm{L}$ hydrochloric acids, which was extracted three times again using $30 \mathrm{~mL}$ of a dichloromethane:ethyl acetate mixture $(1: 1, \mathrm{v}: \mathrm{v})$, and a solid residue was obtained after rotary evaporation at $40^{\circ} \mathrm{C}$. The residue was diluted with $10 \mathrm{~mL}$ of methanol, filtered through a $0.22-\mu \mathrm{m}$ organic filter membrane, and then analyzed via high-performance liquid chromatography (HPLC) (Agilent 6460, USA). $p$-Hydroxybenzaldehyde, vanillin, and syringaldehyde were as standards for the determination of $\mathrm{H}$-lignin, G-lignin, and S-lignin, respectively.

\section{Lignin biosynthesis-related enzyme activity measurements}

First, the crude extracts of the stem were extracted according to the methods in our previous study ${ }^{28}$. Afterward, PAL, C4H, CAD, TAL, and PPO activities were measured using reagent kits, respectively (Keming Biotechnology Co., Ltd., China). Their activity units were expressed in units per gram of FW, and $\mathrm{U}$ indicated the catalytic activity of the enzyme.

\section{PacBio cDNA library construction, sequencing, and data analysis}

Total RNA was extracted using TRIzol reagent (Invitrogen, CA, USA). One cDNA library was constructed by mixing equal amounts of the stems of Xixia Yingxue and Hong Feng at three different developmental stages. First-strand cDNA was synthesized from total RNA using a SMARTer polymerase chain reaction (PCR) cDNA Synthesis Kit (Clontech, USA), and then the first-strand cDNA was synthesized with SMARTScribe Reverse Transcriptase. Subsequently, a large amount of doublestranded cDNA was produced. The cDNA was then measured using Qubit HS (Life Technologies, USA) and an Agilent 2100 Bioanalyzer (Agilent Technologies, CA). Finally, a single molecular real-time (SMRT) bell library was constructed with a PacBio DNA Template Prep Kit, and sequencing reactions were performed using the PacBio RS II Platform (Shenzhen, China).

Raw sequencing data (raw reads) were initially processed with the SMRTlink analysis package. Circular consensus sequences (CCSs) were generated from the initial data. Raw reads with a $5^{\prime}$-primer, $3^{\prime}$-primer, and poly-A tail were defined as the full-length reads. Transcript clusters were found using Iterative Clustering for Error Correction, and LoRDEC software was used to correct nucleotide mismatches. Any redundancy was then deleted by using CD-HIT software ${ }^{30}$ (parameters: -c 0.98, 
-T 6, -G 0, -aL 0.90, -AL 100, -aS 0.98, -AS 30) based on the sequence similarity. Finally, the polished, high-quality, full-length isoforms were compared against the content in public protein databases, such as the nonredundant protein (NR), nucleotide sequence (NT), Protein family (Pfam), euKaryotic Orthologous Groups (KOG), SwissProt, Kyoto Encyclopedia of Genes and Genomes (KEGG), and Gene Ontology (GO) databases.

\section{Illumina cDNA library construction, sequencing, and data} analysis

Eighteen cDNA libraries were constructed using the stems of Xixia Yingxue and Hong Feng at three different developmental stages (three replicates). Purified RNA was fragmented into small pieces with a fragment buffer. The fragmented RNA was then used as a template to synthesize the first-strand cDNA in a first-strand reaction system via PCR, and second-strand CDNA was also synthesized. Magnetic beads were used to purify the reaction products, and then an A-tailing mixture and RNA index adapters were added for end-repair following the Illumina cDNA library construction protocol. PCR was used to amplify the cDNA fragments in conjunction with adapters, and the products were purified by AMPure XP Beads (Beckman, USA). An Agilent 2100 Bioanalyzer (Agilent Technologies, Palo Alto, CA) was used to assess the quality and quantity of the cDNA, after which the qualified library was amplified by an Illumina cBot (Illumina, CA) instrument to generate clusters of the flowcell. The amplified flowcell was subsequently paired-end sequenced on the Illumina HiSeq X-Ten Platform (Shenzhen, China).

The expression level of each gene was calculated by using RSEM ${ }^{31}$ and converted into fragments per kilobase per million fragments (FPKM) according to the read counts. Generally, differential expression analysis was performed by using DESeq2 ${ }^{32}$, with parameters including a fold change $\geq 2.0$ and a false discovery rate $\leq 0.001$.

\section{Gene expression analysis}

Quantitative real-time PCR (qRT-PCR) was used to analyze gene expression levels with a Bio-Rad CFX Connect $^{\mathrm{TM}}$ Optics Module (Bio-Rad, USA), and their values were calculated according to the $2^{-\Delta \Delta \mathrm{Ct}}$ comparative threshold cycle $(\mathrm{Ct})$ method $^{33}$. The specific details of qRT-PCR are available in our previous study ${ }^{28}$. All the primers used are listed in Table S1.

\section{Statistical analysis}

All the data are the average values of at least three replicates and their standard deviations. The variance of the results was analyzed with the SAS/STAT statistical analysis package (version 6.12, SAS Institute, Cary, NC, USA).

\section{Results}

Stem mechanical strength and morphological indices

Two P. lactiflora cultivars with different uprightness were used in this study (Fig. 1a). The stem of Xixia Yingxue began to bend at S2, while the flower drooped significantly at S3. However, the stem of Hong Feng was straight throughout the developmental process. Their stem mechanical strength and morphological indices were then measured (Fig. 1b). The stem mechanical strength of both cultivars gradually increased as the stem developed, and Hong Feng was significantly higher than Xixia Yingxue except at S1. Moreover, the plant height of these two cultivars was essentially the same, while the stem diameter, stem weight, flower diameter, and flower weight were significantly different at S3.

\section{Secondary cell wall}

As shown in Fig. 2a, in addition to the absence of secondary cells in the early stage of stem development (S1), the secondary cell walls of Xixia Yingxue and Hong Feng continued to thicken as the stem developed, the number of thickened cell wall layers increased continuously, and the number of thickened cell wall layers was significantly greater in Hong Feng than in Xixia Yingxue. Moreover, the thickening of the secondary cell walls of the stem was more pronounced under TEM (Fig. 2b). The stems at S1 were primarily composed of parenchyma cells. The secondary cell walls of Xixia Yingxue began to thicken at S2, but the degree of thickening was not yet evident, while the secondary cells of Hong Feng had matured and differentiated at S2. In addition, at S3, the degree of thickening of the secondary cell wall in both cultivars was greater than that observed at S2 and was generally more pronounced in Hong Feng than in the other cultivar.

\section{Lignification of the cell wall and lignin deposition}

Lignification of the cell wall and lignin deposition were further observed using histochemical staining. First, the stem transverse sections were stained using crystal violet, and lignified cell walls were stained violet (Fig. 3a). The degree of lignification of the cell walls of the Hong Feng and Xixia Yingxue stems increased as the stem developed, and the distribution of lignified cell walls increased. When compared with that of Xixia Yingxue, the degree of lignification of Hong Feng stems was relatively higher during stem development.

Wiesner staining is known to react with cinnamaldehyde residues in lignin, and the color intensity was consistent with the lignin content. At S1, the cell walls of both Xixia Yingxue and Hong Feng stems were similarly light in color, with only a small amount of lignin deposition, and there was little difference between them. At S2 and S3, lignin was mainly deposited in the cell walls of sclerenchyma, vascular bundle sheath, and duct. Compared with Xixia Yingxue, Hong Feng presented a greater stained area and a deeper fuchsia color, indicating that the degree of lignin deposition 


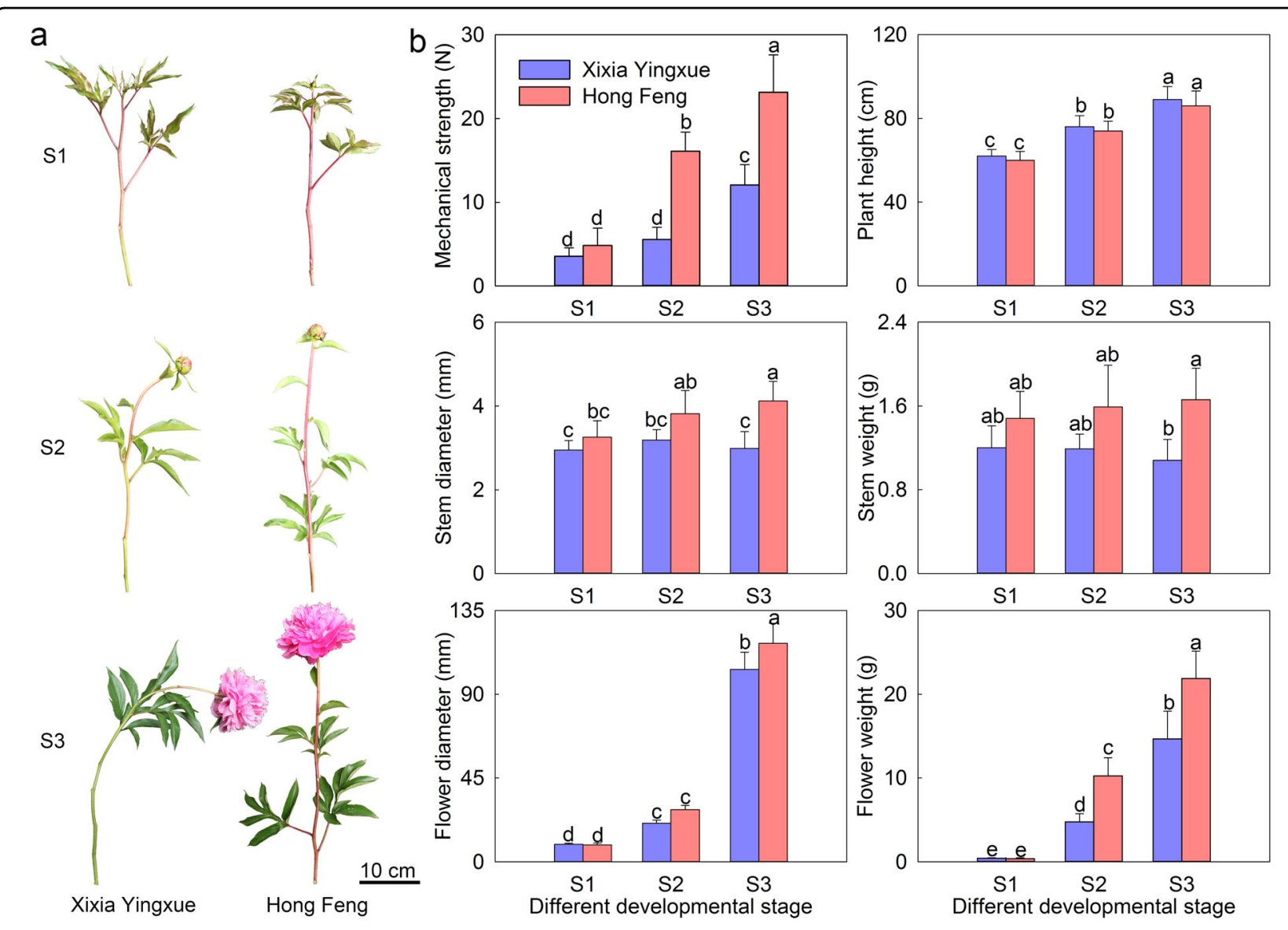

Fig. 1 Images and morphological indices of two P. lactiflora cultivars at three different developmental stages. a Images of two $P$. lactiflora cultivars; b Morphological indices. S1, flower-bud stage; S2, unfolded-petal stage; S3, full-bloom stage. The values represent the means \pm SDs, and different letters indicate significant differences $(P<0.05)$

and the lignin content in Hong Feng were greater than those in Xixia Yingxue (Fig. 3b).

\section{Lignin structure}

FTIR spectrum and 2D-HSQC were used to assess the lignin structure of the stems. The lignin FTIR spectra of Xixia Yingxue and Hong Feng were similar, and the characteristic absorption peaks of the lignin functional groups were primarily concentrated in the range of $1800-900 \mathrm{~cm}^{-1}$ (Fig. 4a). Absorption peaks at $1601 \mathrm{~cm}^{-1}$ and $1512 \mathrm{~cm}^{-1}$ indicated benzene skeleton vibration, absorption peaks at $1329 \mathrm{~cm}^{-1}$ and $1125 \mathrm{~cm}^{-1}$ indicated syringyl ring ( $\mathrm{S}$ unit), absorption peaks at $1269 \mathrm{~cm}^{-1}, 1223 \mathrm{~cm}^{-1}$, absorption peaks at $1033 \mathrm{~cm}^{-1}$ and $834 \mathrm{~cm}^{-1}$ indicated guaiacyl ring (G unit), and the absorption peak at $1165 \mathrm{~cm}^{-1}$ indicated $\mathrm{C}=\mathrm{O}$ stretching vibration of $p$-hydroxyphenyl (the $\mathrm{H}$ unit). In these two cultivars, H-lignin, S-lignin, G-lignin were detected, and the S-lignin, G-lignin, and total lignin contents increased continuously with stem development.

Moreover, Fig. $4 \mathrm{~b}$ showed that the main cross signals in the aromatic ring region $\left(\delta_{\mathrm{C}} / \delta_{\mathrm{H}}\right.$ of $\left.90-160 / 6.0-8.0\right)$ corresponded to the aromatic rings of different lignin units, including syringyl, guaiacyl, and $p$-hydroxyphenyl. A $\mathrm{C}_{2,6}-\mathrm{H}_{2,6}$ correlation was observed in the spectra at $\delta_{\mathrm{C}} /$ $\delta_{\mathrm{H}} 104.1 / 6.74$, representing the $\mathrm{S}$ unit. Signals appearing in the spectra at $\delta_{\mathrm{C}} / \delta_{\mathrm{H}} 111.0 / 7.01,114.4 / 6.73-115.3 / 6.98$, and 119.0/6.82 corresponded to the $\mathrm{C}_{2}-\mathrm{H}_{2}, \mathrm{C}_{5}-\mathrm{H}_{5}$, and $\mathrm{C}_{6}-\mathrm{H}_{6}$ correlations, respectively, representing the $\mathrm{G}$ unit. Additionally, signals of the $\mathrm{C}_{2}-\mathrm{H}_{6}$ correlation, which represented the $\mathrm{H}$ unit, were detected at $\delta 127.8 / 7.22$. It could be seen from the obtained signals that $\mathrm{H}$-lignin was at a low level in Xixia Yingxue and Hong Feng, while S-lignin and G-lignin contents increased significantly during the late stages, and these contents in Hong Feng were higher than those in Xixia Yingxue.

\section{Lignin content and lignin biosynthesis-related enzyme activity}

To confirm the above results, the lignin content of the stems was measured using HPLC (Figs. S1, 5a). The total lignin content of Xixia Yingxue and Hong Feng increased with stem development, and Hong Feng had significantly 


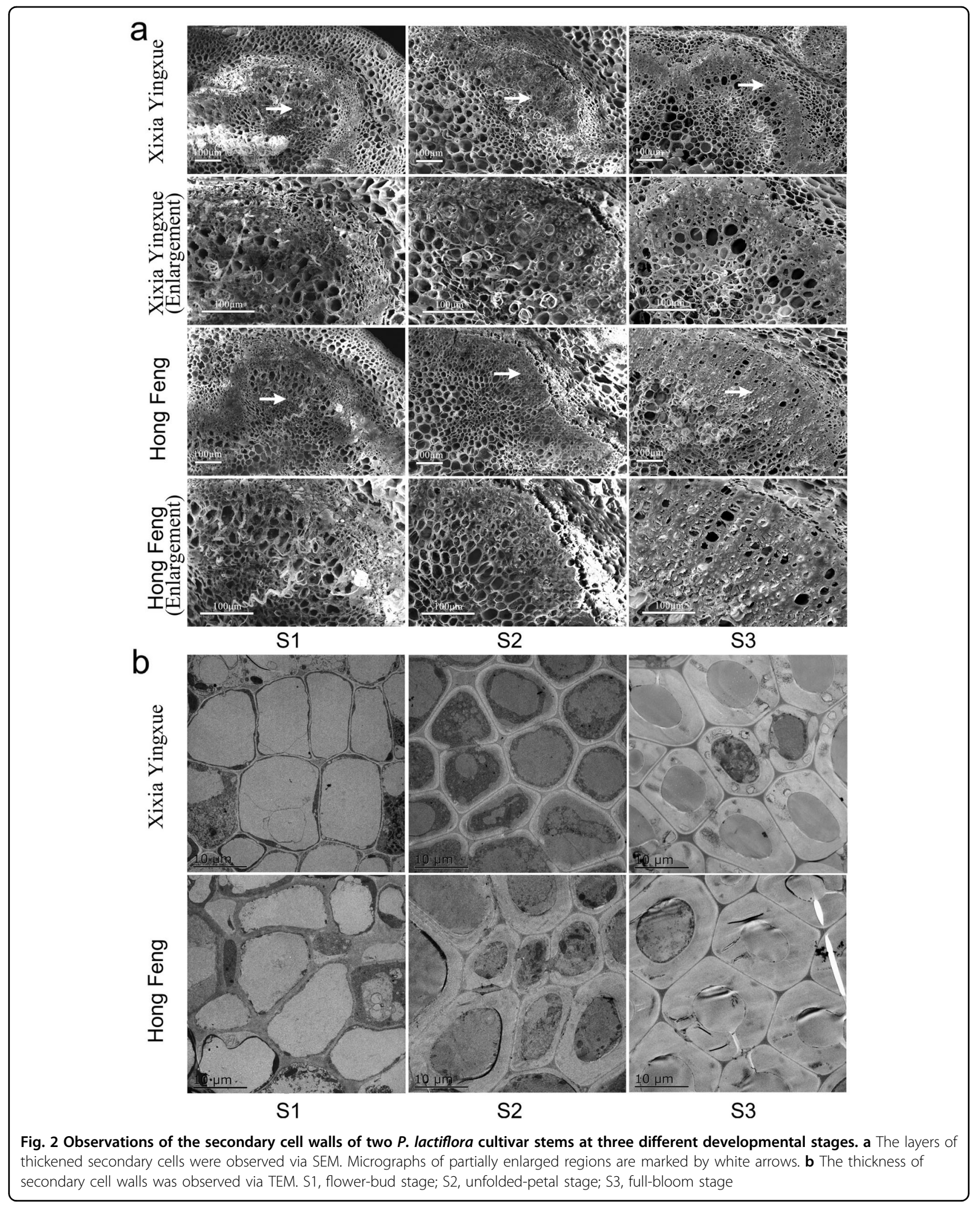



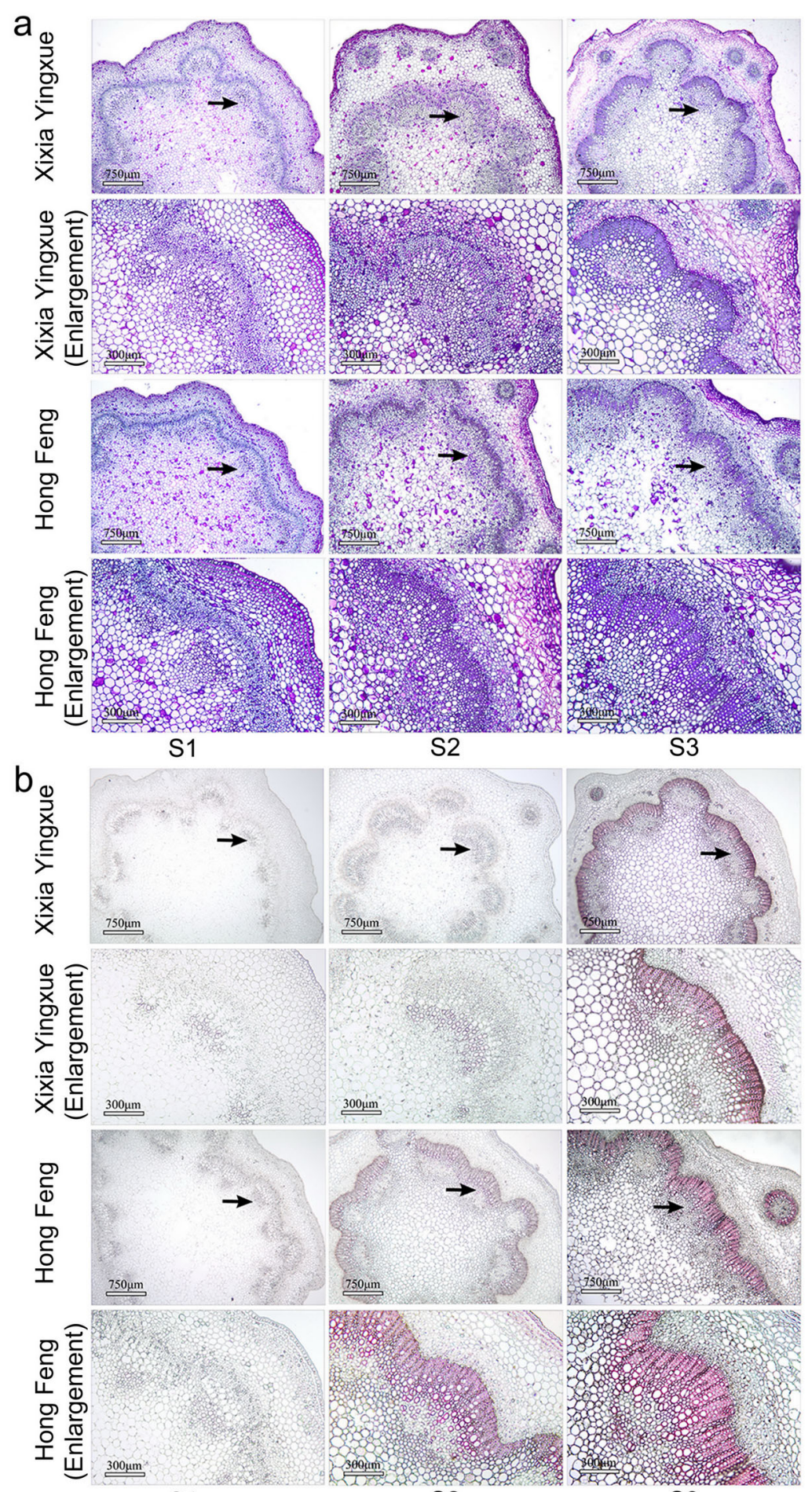

S1

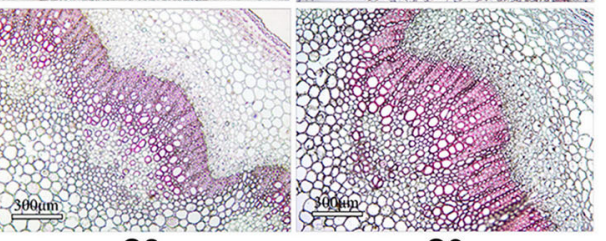

S2

S3

Fig. 3 Histochemical staining of the stems of two P. lactiflora cultivars at three different developmental stages. a Cell wall lignification. $\mathbf{b}$ Lignin deposition. The micrographs of partial enlargement of regions are marked by black arrows. S1, flower-bud stage; S2, unfolded-petal stage; S3, full-bloom stage

higher lignin content than Xixia Yingxue during the late stages. In terms of the contents of the three lignin monomers, it could be clearly seen that S-lignin and G-lignin were the major lignin components of the stems and constantly increased as the stems developed, and Hong Feng had significantly higher lignin contents than Xixia Yingxue in the 

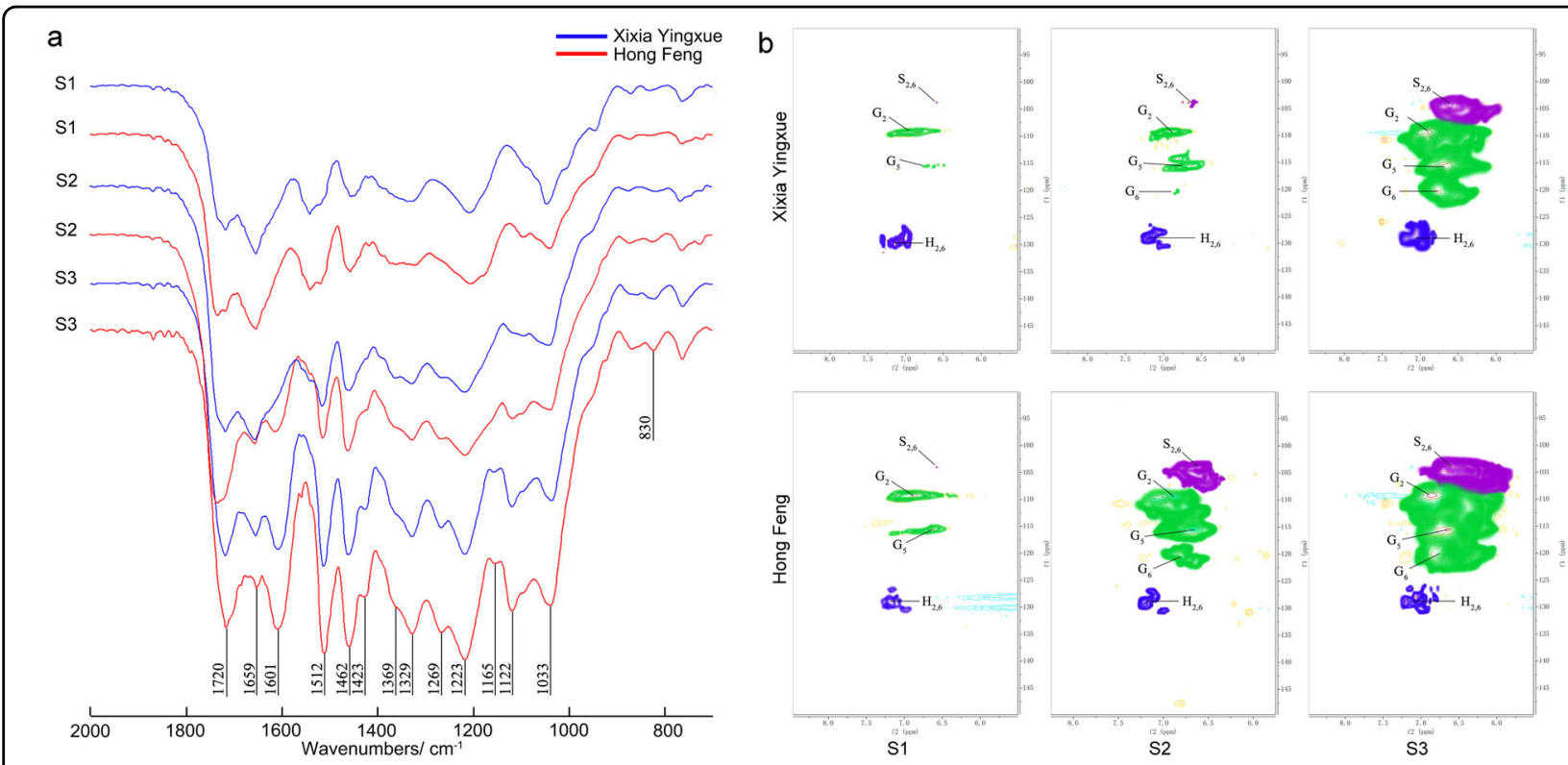

Fig. 4 FTIR spectrum and 2D-HSQC of lignin extracted from two $P$. lactiflora cultivar stem at three different developmental stages. a FTIR spectrum of lignin; b 2D-HSQC results for lignin. $S_{2,6}$ signals represent S-lignin; $G_{2}$, $G_{5}$, and $G_{6}$ signals represent $G$-lignin; and $H_{2,6}$ signals represent H-lignin. S1, flower-bud stage; S2, unfolded-petal stage; S3, full-bloom stage

late stages. The $S / G$ ratio of these two cultivars increased from S1 to S3, and Hong Feng had a consistently higher S/G ratio compared with that of Xixia Yingxue during the stem development.

The activity of six lignin biosynthesis-related enzymes, PAL, C4H, TAL, CAD, PPO and POD, was then analyzed (Fig. 5b). The PAL, C4H, CAD, PPO and POD activities in Hong Feng were higher than those in Xixia Yingxue, and significant differences between Xixia Yingxue and Hong Feng at $\mathrm{S} 3$ were detected for $\mathrm{C} 4 \mathrm{H}, \mathrm{CAD}, \mathrm{PPO}$ and POD activities. However, the TAL activity in these two cultivars decreased, and Hong Feng showed lower TAL activity compared with that of Xixia Yingxue, but the difference was not significant.

\section{Characterization of the full-length transcriptome and functional annotations}

To obtain a better understanding of the molecular mechanisms underlying the lignin regulation of $P$. lactiflora stems, the combined transcript sequencing with SMRT and Illumina sequencing were performed considering no available P. lactiflora genome. A mixture of developing stems of Xixia Yingxue and Hong Feng was used to construct a PacBio cDNA library for full-length transcriptome sequencing. A total of 6,859,926 subreads were generated by SMARTlink software, which was classified into 624,682 nonchimeric CCS reads. Of the CCS reads, there were 456,002 full-length nonchimeric reads, with an average length of $1987 \mathrm{bp}$. Moreover, 191,336 high-quality consensus isoforms (with an average read length of $2108 \mathrm{bp}$ ) were corrected using LoRDEC. Finally, any redundancy was removed by CD-HIT software, and 113,974 corrected fulllength isoforms (with an average read length of $2106 \mathrm{bp}$ ) were ultimately used for subsequent analysis (Table S2).

The full-length isoforms were annotated with seven public reference databases, the NT, NR, GO, KOG, Pfam, KEGG, and SwissProt databases, to obtain comprehensive detailed information on gene function in P. lactiflora. First, the majority of full-length isoforms $(103,413 ; 90.73 \%)$ had similar sequences in the NR database. The matches to other databases were as follows: $90,856(79.72 \%)$ matched to the SwissProt database, 87,807 (77.04\%) matched to the NT database, and 64,611 (56.69\%) matched to the Pfam database using BLAST. All the full-length isoforms were subjected to functional annotation and classification. Most could be annotated by the KEGG, KOG, and GO databases. Based on these results, a total of 105,382 full-length isoforms (92.46\%) were annotated in at least one database, and 42,601 full-length isoforms (37.38\%) were annotated in all databases. Then, we predicted the coding sequence (CDS) and transcription factors (TFs) with coding ability, respectively; the predicted 6009 TFs could be divided into 29 TF families (Fig. 6a-c).

\section{RNA-seq and differential expression analysis based on the full-length transcriptome}

A total of eighteen RNA-seq libraries were constructed for Illumina sequencing. These libraries were generated from the stems of Xixia Yingxue and Hong Feng at three different developmental stages (with three biological replicates). A total of $68.02 \mathrm{M}$ clean reads was obtained on 

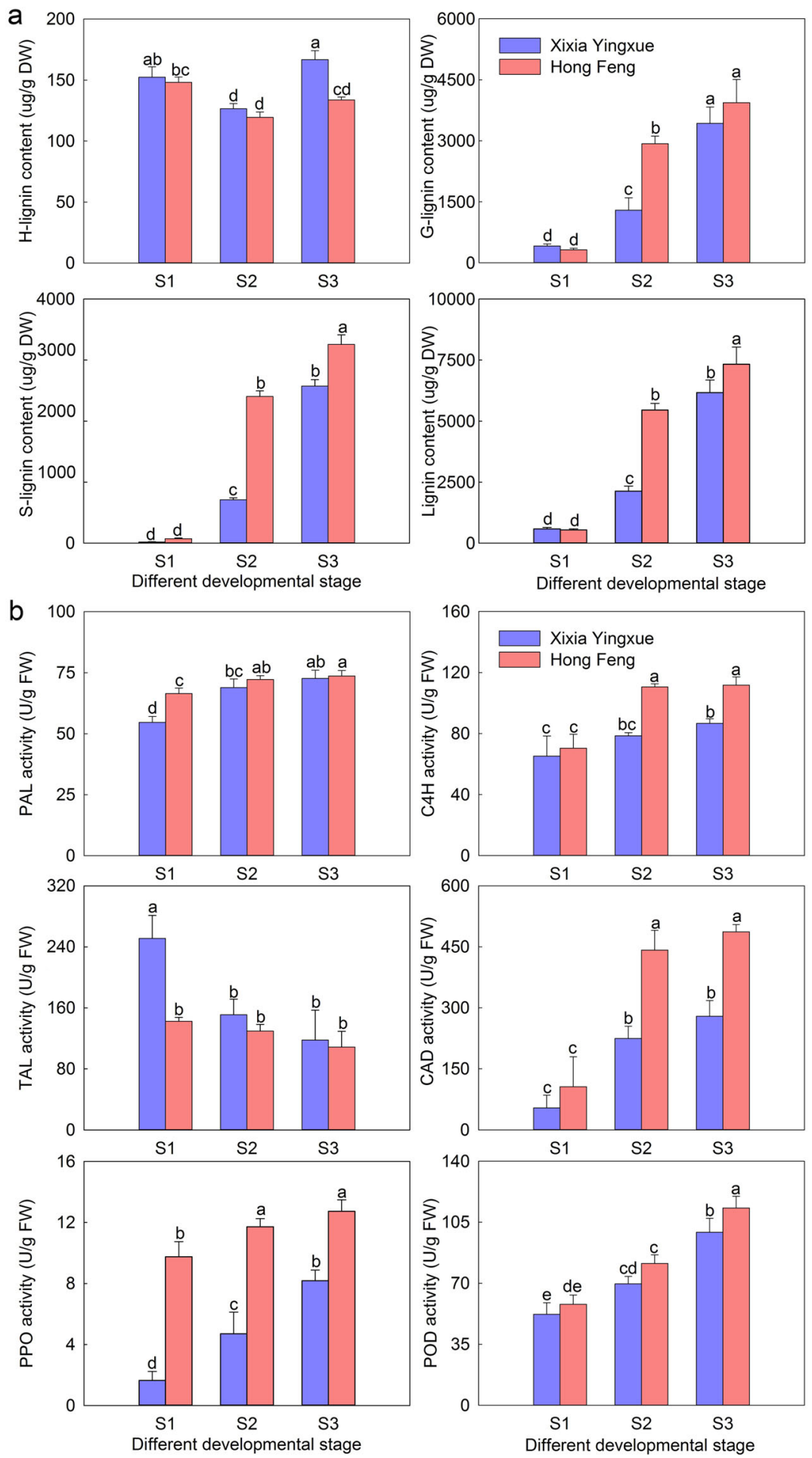

Fig. 5 Lignin content and biosynthesis-related enzyme activities in two P. lactiflora cultivar stem at three different developmental stages. a Lignin content. b Lignin biosynthesis-related enzyme activity. S1, flower-bud stage; S2, unfolded-petal stage; S3, full-bloom stage. The values represent the means \pm SDs, and different letters indicate significant differences $(P<0.05)$ 

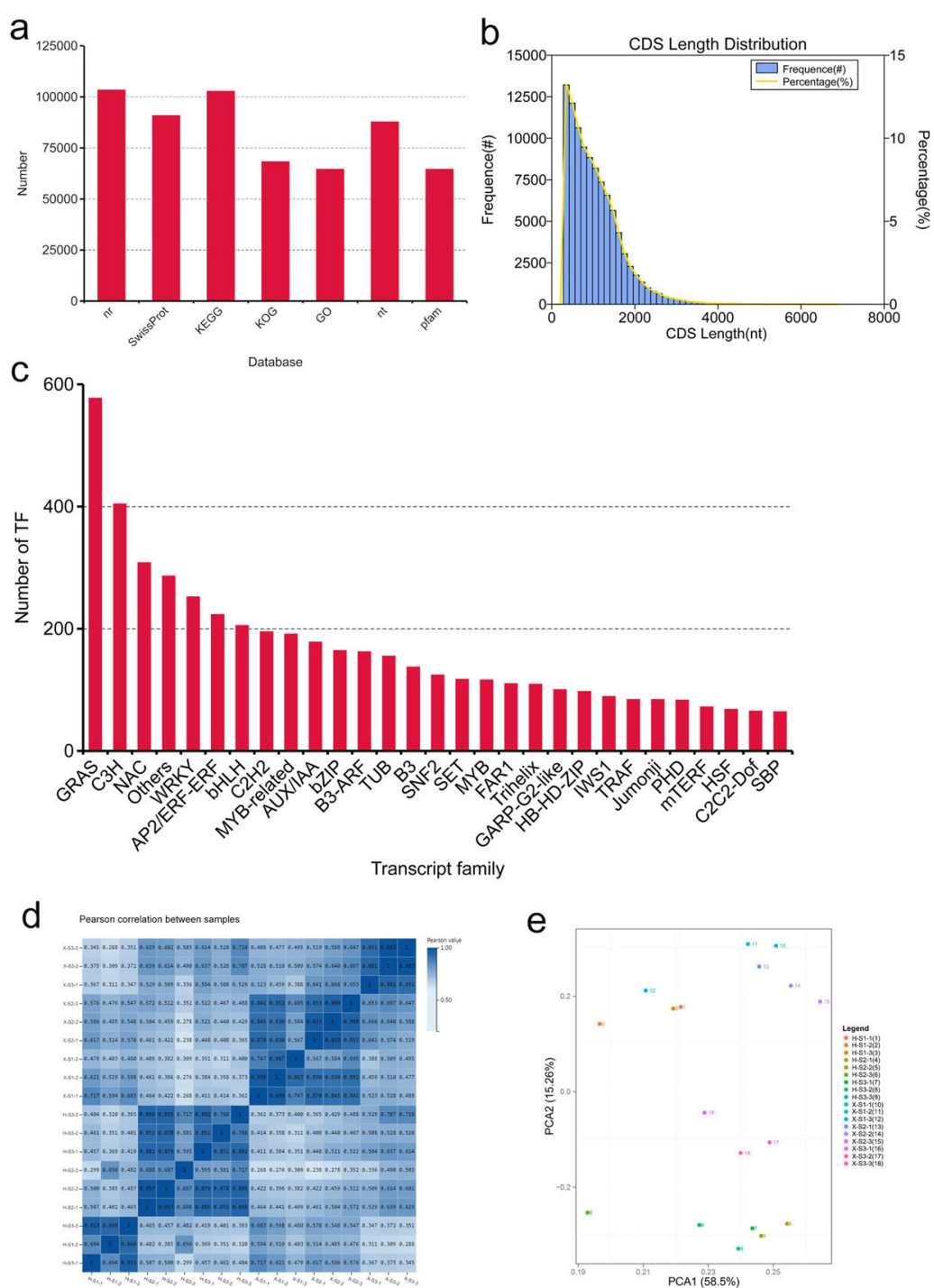

$\mathrm{f}$

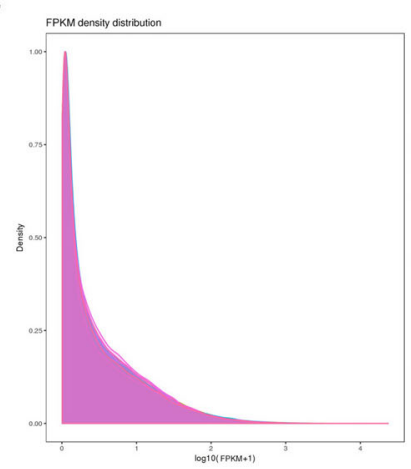

e

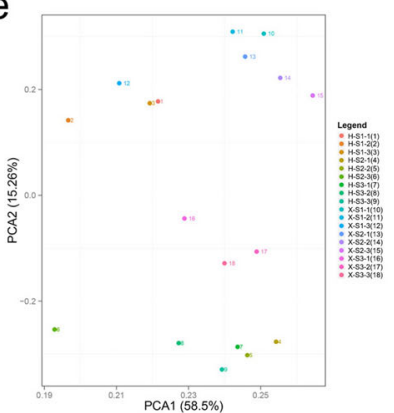

9

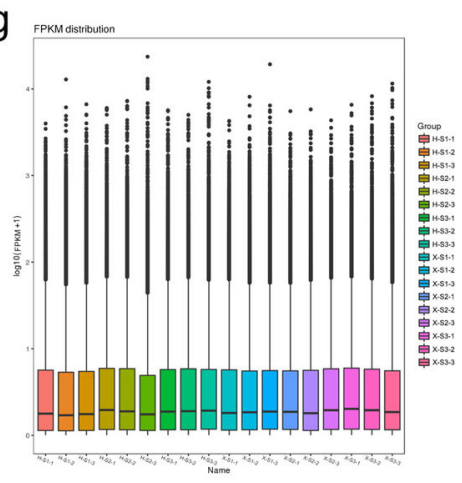

Fig. 6 Gene annotation of full-length isoforms and global gene expression analysis. a Venn diagram of full-length isoforms annotated in the NR, Swissport, KEGG, KOG, GO, NT, and Pfam public databases. b CDS length distribution of full-length isoforms. The $x$-axis represents the CDS length. The $y$-axis represents the number and percentage of the different CDS lengths. c TFs prediction of the full-length isoforms. $\mathbf{d}$ Pearson correlations between eighteen samples based on expression levels. e PCA of eighteen samples based on expression levels. $\mathbf{f}$ Density plot displaying the gene density at different FPKM levels. g Box plot of the FPKM distribution among the eighteen samples. X, Xixia Yingxue; H, Hong Feng; S1, flower-bud stage; S2, unfolded-petal stage; S3, full-bloom stage 
average, which were mapped to the full-length transcriptome using Bowtie2. The average total mapping percentage was $80.25 \%$, and the unique mapping percentage was $9.43 \%$ (Table S3). To confirm the reliability of the RNA-seq data, Pearson correlation analysis, principal component analysis, the FPKM density distribution, and a box plot of all the samples suggested high repeatability of the sequencing results (Fig. $6 \mathrm{~d}-\mathrm{g}$ ).

Pairwise comparisons were conducted to identify differential expression genes (DEGs) among the samples. It was demonstrated that the number of DEGs was highest in Xixia Yingxue-S2 vs. Hong Feng-S2 (36,336: 18,602 upregulated and 17,734 downregulated DEGs), followed by Xixia Yingxue-S1 vs. Hong Feng-S1 (34,703: 16,627 upregulated and 18,076 downregulated DEGs), and lowest in Xixia Yingxue-S3 vs. Hong Feng-S3 (34,512: 16,072 upregulated and 18,440 downregulated DEGs) (Fig. S2). These DEGs were the most responsive to the mechanical strength of the P. lactiflora stems. To validate the accuracy of these DEGs, qRT-PCR was used to analyze the expression levels of nine randomly selected genes, and the results were largely consistent with the RNA-seq data, suggesting that the RNA-seq data were reliable (Fig. S3).

\section{DEGs related to lignin biosynthesis}

A fairly large number of markedly regulated DEGs related to lignin biosynthesis were found, including $P A L, C 4 H$, C3' H, COMT, F5H, 4CL, CCOAOMT, HCT, CCR, CAD, and $P O D$. Subsequently, visual analysis was performed based on their expression levels. The expression levels of these 26 structural genes continuously increased during stem development, and they were higher in Hong Feng than in Xixia Yingxue, which was consistent with change patterns of the lignin content (Fig. 7). In addition, the top $20 \mathrm{TF}$ families with the most DEGs were obtained. Among the TF families whose members presented upregulated expression, the $\mathrm{C} 3 \mathrm{H}$ family contained the most DEGs (64), followed by the MYB family (63 DEGs), and the GRAS family (60 DEGs). A total of 42 upregulated TFs distributed in 17 TF families exhibited increasing expression trends in Xixia Yingxue and Hong Feng during stem development, and higher expression levels were observed in Hong Feng, which was consistent with patterns of lignin biosynthesis (Fig. 8a). Among the TF families whose members presented downregulated expression, the AP2-EREBP family contained the most DEGs (64), followed by the MYB family, with 57 DEGs, and the GRAS family, with 53 DEGs. A total of 14 TFs whose expression was downregulated and that were distributed in $11 \mathrm{TF}$ families showed downward expression trends in Xixia Yingxue and Hong Feng during stem development, and higher expression levels were observed in Xixia Yingxue, which was opposite with patterns of lignin biosynthesis (Fig. 8b).

\section{Co-expression network between lignin-related TFs and structural genes}

To identify genes significantly related to stem strength, we constructed a co-expression network of the above

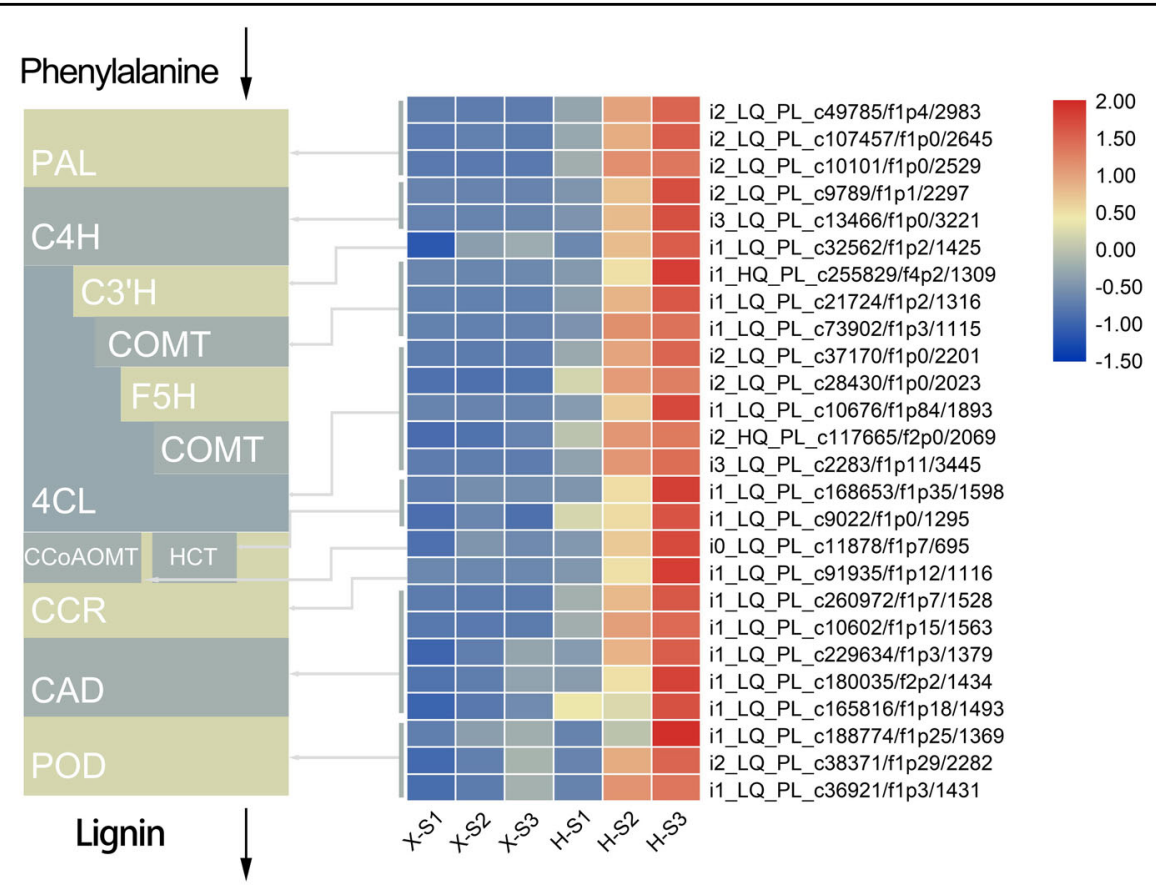

Fig. 7 Differential expression of genes involved in the lignin biosynthesis pathway of the stems of two $P$. lactiflora cultivars at three different developmental stages. X, Xixia Yingxue; H, Hong Feng; S1, flower-bud stage; S2, unfolded-petal stage; S3, full-bloom stage 


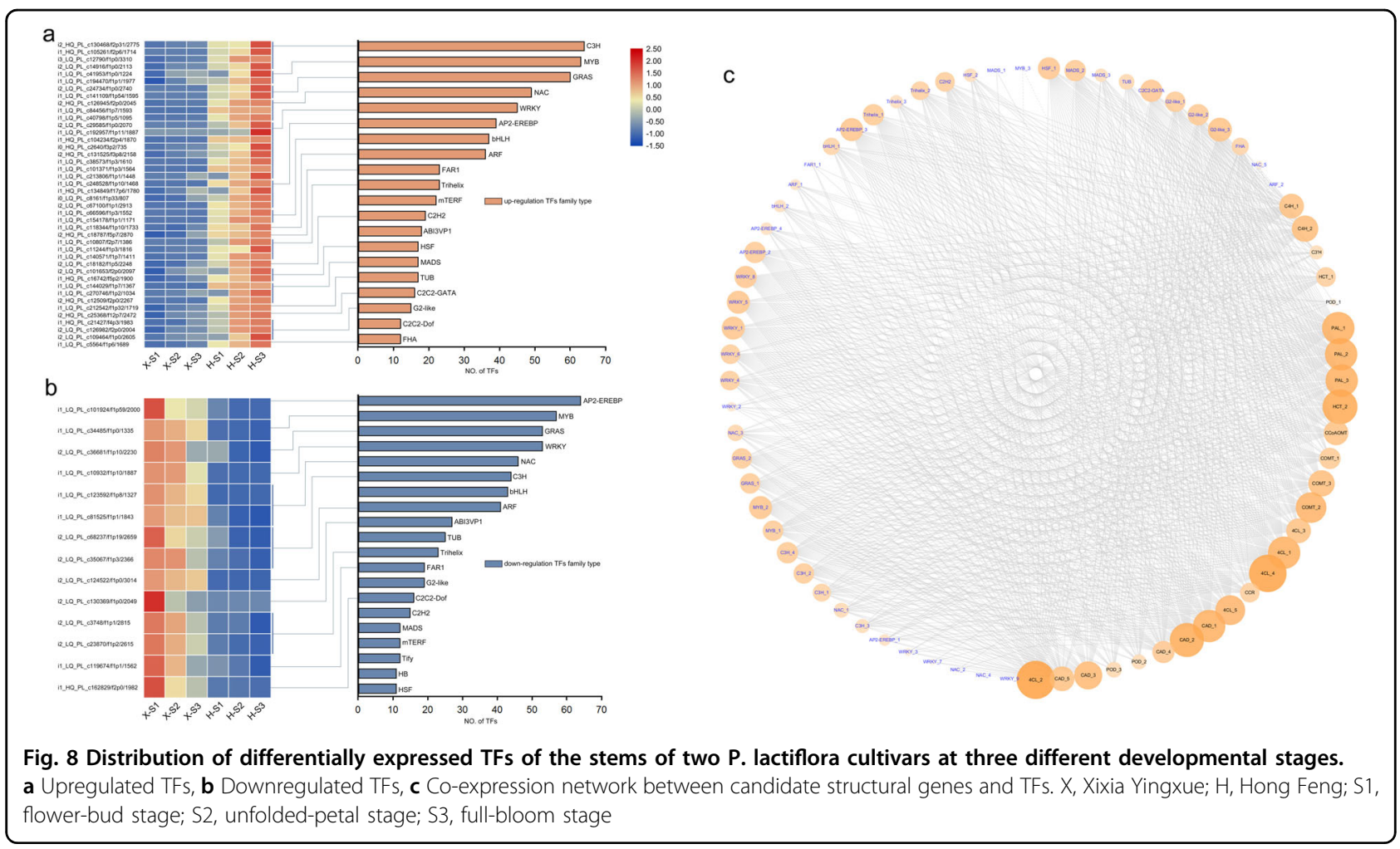

differentially expressed TFs and structural genes (Fig. 8c). The correlation of co-efficient between genes was calculated separately using expression data. We set a threshold of 0.9 for a positive correlation and -0.9 for a negative correlation $(p<0.05)$ and then visualized the network using Cytoscape 3.3.0. A total of 47 TFs (42 upregulated ones, 5 downregulated ones) were believed to have a high degree of co-expression relationship with 26 structural genes. The darker the color of the node, the larger radius indicated the stronger connectivity, which meant that the corresponding genes were more important.

Among the structural genes, 4CL_2 (i2_LQ_PL_c28430/ f1p0/2023) was linked to the most numbers of TFs, followed by 4CL_4 (i2_HQ_PL_c117665/f2p0/2069), HCT_2 (i1_LQ_PL_c9022/f1p0/1295) and CAD_2 (i1_LQ_PL_c1 0602/f1p15/1563), which were all downstream genes that catalyzed lignin biosynthesis. In the upstream of lignin metabolism, the identified $P A L_{-} 1$ (i2_LQ_PL_c49785/ f1p4/2983), PAL_2 (i2_LQ_PL_c107457/f1p0/2645), $P A$ L_3 (i2_LQ_PL_c10101/f1p0/2529), C4H_1 (i2_LQ_PL c9789/f1p1/2297), and C4H_2 (i3_LQ_PL_c13466/f1p0/ 3221) genes had high connectivity with TFs. The connected TFs included 39 TFs belonging to 16 TFs families, and the WRKY family TFs shared the most connections with the 5 structural genes. Other identified important TFs included 3 NAC family TFs, 2 MYB family TFs, and 4 $\mathrm{C} 3 \mathrm{H}$ family TFs, which were thought co-expressed with upstream structural genes (Table S4). At the same time,
NAC_5 (i1_LQ_PL_c81525/f1p1/1843) was identified as the only correlated TF, which was linked to $P A L_{-} 3$, showing the opposite expression trend with it.

\section{Discussion}

P. lactiflora stem mechanical strength is closely related to related indices of stems and flowers, and significantly positive correlations between stem diameter, flower diameter, and flower weight and stem mechanical strength have been found ${ }^{22}$. In this study, the stem mechanical strength, stem diameter, flower diameter, and flower weight of Xixia Yingxue and Hong Feng increased with plant development, and the stem mechanical strength and stem diameter of Hong Feng were always higher than those of Xixia Yingxue, which was in agreement with the results of a previous study ${ }^{28}$, suggesting that stem diameter could be used as a visual indicator of stem mechanical strength in P. lactiflora. Moreover, compared with those of Xixia Yingxue, the flower diameter and flower weight of Hong Feng were higher at S2 and S3, and these results were largely consistent with the trends of the stem mechanical strength, which might be because the rapid development of flowers during the middle and late stages required higher stem mechanical strength to provide support. A previous study in rice showed that the gradual accumulation and output of nutrients occurred simultaneously during the process of stem formation and senescence; specifically, when the accumulation and 
output were the same and equilibrium had been reached, the nutrients of the stem were sufficient, and the stem mechanical strength was maximized ${ }^{34}$. The nutrients in P. lactiflora stems had accumulated to a level that was at or greater than the output level from S1 to S3, the stem diameter increased, and the stem mechanical strength improved continuously, thus providing mechanical support for the developing flowers.

Stem mechanical strength is closely related to stem anatomical structure. In barley, stems with thicker mechanical structures had greater numbers of cell layers and stronger lodging resistance ${ }^{35}$. The stems of lodgingresistant rice cultivars had many structural advantages, such as a thickened stem wall, well-developed mechanical tissue, and a large number of vascular bundles ${ }^{36}$. In P. lactiflora, the number of vascular bundles in the stems of Hongyan Zhenghui increased, the cell walls of thickwalled tissue thickened, and the proportion of vascular bundles and pith increased with plant development ${ }^{22}$. In this study, the secondary cell walls of the Xixia Yingxue and Hong Feng stem continued to thicken with the development of the plants, and the number of layers with thickened secondary cell walls also increased; moreover, the number of layers was significantly higher in Hong Feng than in Xixia Yingxue. When P. lactiflora was treated with calcium, the stems with increased mechanical strength had significantly thickened secondary cell walls and increased cell layers, indicating that secondary cells played an important role in the formation of stem mechanical strength ${ }^{25}$. In addition, differences in the lignification of the cell walls also have a great impact on stem mechanical strength ${ }^{35}$. In this study, there was a difference in the lignification of cell walls in P. lactiflora stems, and the lignification of Hong Feng was higher than that of Xixia Yingxue during stem development. Further observations of the distribution of lignin showed that the lignin coloring intensity gradually increased with stem development, and the coloring range of Hong Feng was greater than that of Xixia Yingxue, which was consistent with the lignin content measurements. In addition, lignin content is closely related to the activity of related enzymes in the lignin biosynthesis pathway. In common buckwheat, the lignin content is significantly positively correlated with PAL, 4CL, CAD and POD activities ${ }^{37,38}$. In the present study, the activities of PAL, C4H, CAD, PPO and POD were higher in Hong Feng than in Xixia Yingxue during stem development, and the activity of all tended to increase, which was in agreement with the obtained lignin content. The trend in TAL activity was the exact opposite of the trend in lignin content in Hong Feng and Xixia Yingxue, indicating that TAL was not important to the lignin biosynthesis of $P$. lactiflora stem.

There are three types of lignin-H-lignin, G-lignin, and S-lignin, and the types and contents of lignin monomers in different plant species are not consistent. G-lignin and $\mathrm{S}$-lignin could be detected in pear fruits ${ }^{39}$, and the lignin from macaúba stems was enriched in $S$-lignin ${ }^{40}$. In this study, FTIR analysis showed that the characteristic peaks of syringyl, guaiacyl, and $p$-hydroxyphenyl structural units were clearly present, and 2D-HSQC analysis also detected $\mathrm{H}$-lignin, G-lignin, and S-lignin, which indicated that the lignin of the stems comprised G-lignin, S-lignin and $\mathrm{H}$-lignin in P. lactiflora. Quantitative analysis of the lignin revealed that G-lignin and S-lignin were the main lignin monomers of $P$. lactiflora stems, and their contents were generally higher in Hong Feng than in Xixia Yingxue. The S/G ratio of these two cultivars increased from S1 to S3, and the S/G ratio of Hong Feng was always higher than that of Xixia Yingxue. Similarly, Pramod et al. observed decreases in the contents of S-lignin and G-lignin in subabul tension wood, and the $S / G$ ratio was significantly lower than that of its opposite wood, which was in agreement with the results of this study ${ }^{41}$. These results indicated that S-lignin played an important role in the formation of P. lactiflora stem mechanical strength, which was in agreement with previous studies showing that S-lignin mainly played a role in mechanical support ${ }^{15,42}$.

Because of its large genome and high heterozygosity, genome sequencing has not yet been completed for $P$. lactiflora, which has made the study of this species increasingly difficult. In recent years, third-generation fulllength transcriptome sequencing based on PacBio SMRT sequencing technology has facilitated molecular biology research for plants lacking genomic information, and this technique has been used in Coptis deltoidea ${ }^{43}$, Crocus sativus $^{44}$, and Olea europaea ${ }^{45}$. In this study, a mixture of stems of Xixia Yingxue and Hong Feng at different developmental stages was used to construct a PacBio cDNA library for full-length transcriptome sequencing, and 113,974 full-length isoforms with an average read length of 2106 bp were obtained, laying a foundation for identifying excellent genes that regulated the mechanical strength of P. lactiflora stems. On this basis, RNA-seq was then used to sequence stems of Xixia Yingxue and Hong Feng at three different developmental stages, and a total of $68.02 \mathrm{M}$ clean reads were obtained on average, with an average total mapping percentage was $80.25 \%$. In addition, a large number of DEGs between these two cultivars were obtained, and these results were confirmed by qRT-PCR.

Genes in the lignin biosynthetic pathway can be divided into three categories. One category contains $P A L, C 4 H$, and $4 C L$ in the phenylpropanoic acid pathway, and their expression levels are significantly correlated with lignin content ${ }^{46}$. In transgenic plants, low expression levels of these three genes resulted in decreases in lignin content ${ }^{47,48}$. The second category is the lignin monomer biosynthesis-related genes, including $H C T, C 3^{\prime} H$, CCOAOMT, COMT, and $F 5 H$, which influence lignin 
monomer biosynthesis, determine the proportion of various monomers in lignin, and thus affect the total lignin content to some extent ${ }^{49}$. In jute or Arabidopsis, the repression of COMT or $F 5 H$ expression resulted in significant decreases in S-lignin ${ }^{50,51}$. Moreover, downstream of the lignin biosynthetic pathway, some genes involved in lignin monomer biosynthesis and polymerization, such as $C C R, C A D$, and $P O D^{49}$, were significantly positively correlated with lignin content ${ }^{52}$. In the present study, 26 DEGs were identified as being related to lignin biosynthesis, including $3 P A L, 2 C 4 H, 1 C^{\prime} H, 3 C O M T, 5$ 4CL, 1 CCoAMOT, $2 H C T, 1 C C R, 5 C A D$, and $3 P O D$ genes. The expression levels of these DEGs related to lignin biosynthesis tended to increase in the stems of Xixia Yingxue and Hong Feng at different developmental stages, and the expression levels of these genes were always higher in Hong Feng than in Xixia Yingxue, which was consistent with the observed changes in lignin content. These genes could provide a foundation for improving stem mechanical strength via genetic engineering. In addition, studies in model plant species have shown that lignin biosynthesis is regulated by multiple TFs, such as the MYB and NAC families ${ }^{53}$. In this study, the expression levels of 42 upregulated TFs distributed in $17 \mathrm{TF}$ families were consistent with the patterns of lignin biosynthesis, while the expression levels of 14 downregulated TFs distributed in $11 \mathrm{TF}$ families were opposite with patterns of lignin biosynthesis.

To further clarify the relationships between TFs and structural genes, we constructed a co-expression network between different genes based on the RNA-seq data. In this network, we identified multiple downstream genes that catalyzed lignin biosynthesis, including 4CL_2, $4 C L \_4, H C T \_2$, and $C A D \_2$. We focused on the differentially expressed structural genes upstream of lignin metabolism, and identified a total of 5 structural genes (PAL_1, PAL_2, PAL_3, C4H_1, and C4H_2) that were highly co-expressed with a large number of TFs. These genes formed 164 co-expression relationships with $39 \mathrm{TFs}$ from 16 TF families. At the transcriptional level, multiple TF families regulate lignin biosynthesis and form complex transcriptional regulatory networks ${ }^{53}$. The NAC family acts as the first-level master switches to control the expression of downstream $\mathrm{TFs}^{54,55}$. The second-level of regulators includes many MYB TFs and zinc-finger $\mathrm{TFs}^{56}$. In this study, a total of three NAC family TFs, two MYB family TFs, and four $\mathrm{C} 3 \mathrm{H}$ family $\mathrm{TFs}$ were identified. The final results showed that this TFs might regulate stem lignification via these five structural genes. Among them, $N A C \_5$ was the only downregulated TF, which was connected to the upstream $P A L \_3$. The predicted relationships between TFs and structural genes provided us with very useful information to help us analyze the underlying factors of the stem strength diversity among different cultivars in more depth. Overall, this differentially expressed TFs could be further analyzed via biochemical experiments to characterize their gene functions underlying lignin biosynthesis in P. lactiflora stems.

\section{Conclusions}

P. lactiflora stems accumulate high amounts of lignin during their development, and this accumulated lignin provides mechanical support to the stems. The thickening of the secondary cell wall, number of thickened secondary cell wall layers, lignin deposition, S-lignin content, and lignin biosynthesis-related enzyme activities were all positively correlated with stem mechanical strength. By comparing the transcriptome profiles of two P. lactiflora cultivar stems at different stages, key genes involved in the lignin biosynthetic pathway were identified, and these genes contributed directly to the regulation of lignification in P. lactiflora stems. Moreover, the expression profiles of a number of differentially expressed TFs, including three NAC family TFs, two MYB family TFs, and four $\mathrm{C} 3 \mathrm{H}$ family TFs were highly correlated with the expression profiles of key lignin biosynthesis-related genes. The findings of this work provide useful information for understanding the formation of P. lactiflora stem strength.

\begin{abstract}
Acknowledgements
This work was supported by the Natural Science Foundation of China (31772341 and 31972448), the Fifth Phase of the "Project 333" Science Funding Program of Jiangsu Province (BRA2019084), and the Young Talent Support

Project of Jiangsu Provincial Association for Science and Technology.

\section{Author contributions}

J.T. and D.Z. designed the experiments. X.X., W.S., Y.L., and Y.T. performed the experiments. D.Z., Y.L., X.X., and W.S. analyzed the data and wrote the paper. All the authors have carefully read and approved the final paper.
\end{abstract}

Conflict of interest

The authors declare that they have no conflict of interest.

Supplementary Information accompanies this paper at (https://doi.org/ 10.1038/s41438-020-00451-5).

Received: 13 July 2020 Revised: 19 November 2020 Accepted: 20 November 2020

Published online: 28 December 2020

\footnotetext{
References

1. Perik, R. R. J., Raze, D., Harkema, H., Zhong, Y. \& van Doorn, W. G. Bending in cut Gerbera jamesonii flowers relates to adverse water relations and lack of stem sclerenchyma development, not to expansion of the stem central cavity or stem elongation. Postharvest Biol. Technol. 74, 11-18 (2012).

2. Kamran, M. et al. Application of paclobutrazol: a strategy for inducing lodging resistance of wheat through mediation of plant height, stem physical strength, and lignin biosynthesis. Environ. Sci. Pollu. Res. 25, 29366-29378 (2018).

3. Jin, H. \& Kwon, M. Mechanical bending-induced tension wood formation with reduced lignin biosynthesis in Liriodendron tulipifera. J. Wood Sci. 55, 401-408 (2009).

4. Xia, X., Tang, Y., Tao, J. \& Zhao, D. Formation and regulation of ornamental plant stem strength. Plant Physiol. J. 5, 347-354 (2018). (in Chinese).

5. $\mathrm{Hu}, \mathrm{K}$. et al. Improvement of multiple agronomic traits by a disease resistance gene via cell wall reinforcement. Nat. Plants 3, 17009 (2017).
} 
6. Wójtowicz, T., Grabowska-Joachimiak, A. \& Zieliński, A. Analysis of morphoanatomical stem properties determining its mechanical strength in selected rye cultivars. Int. Agrophys. 34, 123-131 (2020).

7. Liu, B. \& Koc, A. B. Mechanical properties of switchgrass and miscanthus. Trans. ASABE 60, 581-590 (2017).

8. Hussain, S. et al. Effects of lignin, cellulose, hemicellulose, sucrose and monosaccharide carbohydrates on soybean physical stem strength and yield in intercropping. Photochem. Photobiol. Sci. 19, 462 (2020).

9. Ragauskas, A. J. et al. Lignin valorization: improving lignin processing in the biorefinery. Science 344, 1246843 (2014).

10. $L i, Y$. et al. Acid detergent lignin, lodging resistance index, and expression of the caffeic acid O-methyltransferase gene in brown midrib-12 sudangrass. Breed. Sci. 65, 291-297 (2015).

11. LV, G. S. et al. The anatomy and physiology of spray cut chrysanthemum pedicels, and expression of a caffeic acid 3-O-methyltransferase homologue. Postharvest Biol. Technol. 60, 244-250 (2011).

12. Sreeja, R. et al. Association of lignin and FLEXIBLE CULM 1 (FC1) ortholog in imparting culm strength and lodging resistance in kodo millet (Paspalum scrobiculatum L.). Mol. Breed. 36, 149 (2016).

13. Li, X. et al. FLEXIBLE CULM 1 encoding a cinnamyl-alcohol dehydrogenase controls culm mechanical strength in rice. Plant Mol. Biol. 69, 685-697 (2009).

14. Liu, Q. Q., Luo, L. \& Zheng, L. Q. Lignins: biosynthesis and biological functions in plants. Int. J. Mol. Sci. 19, 335 (2018).

15. Zheng, M. J. et al. Manipulation of lignin metabolism by plant densities and its relationship with lodging resistance in wheat. Sci. Rep. 7, 41805 (2017).

16. Wang, G. L. et al. Transcriptome based identification of genes revealed differential expression profiles and lignin accumulation during root development in cultivated and wild carrots. Plant Cell Rep. 35, 1743-1755 (2016).

17. Peng, D. L. et al. Lodging resistance of winter wheat (Triticum aestivum L.): lignin accumulation and its related enzymes activities due to the application of paclobutrazol or gibberellin acid. Field Crop. Res. 157, 1-7 (2014).

18. Liu, W. G. et al. Shade stress decreases stem strength of soybean through restraining lignin biosynthesis. J. Integr. Agric. 18, 43-53 (2019).

19. Kamran, M. et al. Mepiquat chloride application increases lodging resistance of maize by enhancing stem physical strength and lignin biosynthesis. Field Crop Res. 224, 148-159 (2018).

20. Li, C., Sun, Y., Zhao, D. \& Tao, J. Relationship between mechanical strength and morphological index of inflorescence stem of herbaceous peony (Paeonia lacflora Pall.). Acta Agric. Zhejiangensis 27, 182-188 (2015). (in Chinese).

21. Li, C., Sun, Y., Zhao, D., Feng, L. \& Tao, J. Relationship between inflorescence stem mechanical strength and some elements contents of herbaceous peony (Paeonia lactiflora Pall.). Southwest China J. Agric. Sci. 29, 1214-1218 (2016). (in Chinese).

22. Zhao, D., Han, C., Tao, J., Wang, J. \& Hao, Z. Effects of inflorescence stem structure and cell wall compositions on mechanical strength of inflorescence stem in herbaceous peony. Int. J. Mol. Sci. 13, 4993-5009 (2012).

23. Zhao, L., Liu, A., Zhang, J., Han, J. \& Liu, Y. Study on the stem orthostatic performance of Paeonia lactiflora under facility cultivation. Acta Agric. Zhejiangensis 27, 769-775 (2015). (in Chinese).

24. Wan, Y. et al. Transcriptome and weighted correlation network analyses provide insights into inflorescence stem straightness in Paeonia lactiflora. Plant Mol. Biol. 102, 239-252 (2020).

25. Zhao, D. et al. Integration of transcriptome, proteome, and metabolome provides insights into how calcium enhances the mechanical strength of herbaceous peony inflorescence stems. Cells 8, 102 (2019).

26. Tang, Y., Zhao, D., Meng, J. \& Tao, J. EGTA reduces the inflorescence stem mechanical strength of herbaceous peony by modifying secondary wall biosynthesis. Hortic. Res. 6, 36 (2019).

27. Zhao, D., Hao, Z., Tao, J. \& Han, C. Silicon application enhances mechanical strength of the inflorescence stem in herbaceous peony (Paeonia lactiflora Pall.). Sci. Hortic. 151, 165-172 (2013).

28. Zhao, D., Shi, W., Xia, X., Tang, Y. \& Tao, J. Microstructural and lignin characteristics in herbaceous peony cultivars with different stem strengths. Postharvest Biol. Technol. 159, 111043 (2020).

29. Li, Y. Z., Jia, H. Z., Ruxianguli, R., Yin, H. Q. \& Zhang, Q. S. Extraction of lignin from wheat straw by catalysts in 1, 4-butanediol medium under atmospheric pressure. Bioresources 10, 1085-1098 (2015).

30. Fu, L. M., Niu, B. F., Zhu, Z. W., Wu, S. T. \& Li, W. Z. CD-HIT: accelerated for clustering the next generation sequencing data. Bioinformatics 28, 3150-3152 (2012).
31. Li, B. \& Dewey, C. N. RSEM: accurate transcript quantification from RNA-Seq data with or without a reference genome. BMC Bioinform. 12, 323 (2011).

32. Love, M. I., Huber, W. \& Anders, S. Moderated estimation of fold change and dispersion for RNA-seq data with DESeq2. Genome Biol. 15, 550 (2014).

33. Schmittgen, T. D. \& Livak, K. J. Analyzing real-time PCR data by the comparative CT method. Nat. Protoc. 3, 1101 (2008)

34. Lin, X. Effect of Silicon on the Strength of Rice Stem. (Master's Thesis, Sichuan Agricultural University, Shanghai, China, 2010). (in Chinese).

35. Zha, S. The relationship between morphological characteristics and anatomical structure and lodging of the barley stem. Tibet. J. Agric. Sci. 39, 10-14 (2017). (in Chinese).

36. Yang, $Y$. et al. Relationship between anatomic structure of the stem and lodging resistance of rice. Guihaia 32, 834-839 (2012). (in Chinese).

37. Chen, $X$. et al. Effects of uniconazole or gibberellic acid application on the lignin metabolism in relation to lodging resistance of culm in common buckwheat (Fagopyrum esculentum M.). J. Agron. Crop Sci. 20, 414-423 (2018). (in Chinese).

38. Wang, C. et al. Effects of uniconazole on the lignin metabolism and lodging resistance of culm in common buckwheat (Fagopyrum esculentum M.). Field Crops Res. 180, 46-53 (2015).

39. Zhang, L. M., Kamitakahara, H., Murayama, H., Ohsako, T. \& Itai, A. The analysis of fruit lignin content, composition and linkage types in pear cultivars and related species. J. Agric. Food Chem. 68, 2493-2505 (2020).

40. Rencoret, J. et al. Variability in lignin composition and structure in cell walls of different parts of macaúba (Acrocomia aculeata) palm fruit. J. Agric. Food Chem. 66, 138-153 (2018).

41. Pramod, S., Rao, K. S. \& Sundberg, A. Structural, histochemical and chemical characterization of normal, tension and opposite wood of Subabul (Leucaenaleucocephala (lam.) De wit.). Wood Sci. Technol. 47, 777-796 (2013).

42. Wang, M. X. et al. A wheat caffeic acid 3-O-methyltransferase TaCOMT-3D positively contributes to both resistance to sharp eyespot disease and stem mechanical strength. Sci. Rep. 8, 6543 (2018)

43. Zhong, F., Huang, L., Qi, L., Ma, Y. \& Yan, Z. Full-length transcriptome analysis of Coptis deltoidea and identification of putative genes involved in benzylisoquinoline alkaloids biosynthesis based on combined sequencing platforms. Plant Mol. Biol. 102, 477-499 (2020)

44. Yue, J. et al. Full-length transcriptome sequencing provides insights into the evolution of apocarotenoid biosynthesis in Crocus sativus. Comput. Struct. Biotechnol. J. 18, 774-783 (2020).

45. Rao, G., Zhang, J., Liu, X. \& Luo, Y. Identification of putative genes for polyphenol biosynthesis in olive fruits and leaves using full-length transcriptome sequencing. Food Chem. 300, 125246 (2019).

46. Yoon, J., Choi, H. \& An, G. Roles of lignin biosynthesis and regulatory genes in plant development. J. Integr. Plant Biol. 57, 902-912 (2015).

47. Li, Y. et al. BRITTLE CULM1, which encodes a COBRA-like protein, affects the mechanical properties of rice plants. Plant Cell 15, 2020-2031 (2003).

48. Sewalt, V. et al. Reduced lignin content and altered lignin composition in transgenic tobacco down-regulated in expression of I-phenylalanine ammonia-lyase or cinnamate 4-hydroxylase. Plant Physiol. 115, 41-50 (1997).

49. Zhao, Q. \& Dixon, R. A. Transcriptional networks for lignin biosynthesis: more complex than we thought. Trends Plant Sci. 16, 227-233 (2011)

50. Shafrin, F. et al. Modification of monolignol biosynthetic pathway in jute: different gene, different consequence. Sci. Rep. 7, 39984 (2017).

51. Gallego-Giraldo, L. et al. Elicitors and defense gene induction in plants with altered lignin compositions. N. Phytol. 219, 1235-1251 (2018).

52. Shan, L. L. et al. Characterization of CDNAs associated with lignifications and their expression profiles in loquat fruit with different lignin accumulation. Planta 227, 1243-1254 (2008).

53. Rao, X. \& Dixon, R. A. Current models for transcriptional regulation of secondary cell wall biosynthesis in grasses. Front. Plant Sci. 9, 399 (2018).

54. Huang, C. \& Li, L. G. Research progress on regulation of plant secondary cell wall thickening. Plant Physiol. J. 52, 8-18 (2016). (in Chinese).

55. Zhu, X. B., Zhang, G. F. \& Chen, P. Research progress in the transcriptional regulation of secondary cell wall thickening. Plant Physiol. J. 53, 1598-1608 (2017). (in Chinese).

56. Cassan-Wang, $\mathrm{H}$. et al. Identification of novel transcription factors regulating secondary cell wall formation in Arabidopsis. Front. Plant Sci. 4, 189 (2013) 